"If you put your foot off the gas one day a week but then, six days a week, you have it pounded to the metal, you are not necessarily doing yourself a whole lot of good," says Freedland.

A more sensible approach, he suggests, might be to compensate for the occasional indulgence by eating slightly less during the remainder of the week. "You want to have cake on Friday night because it's your birthday? Have the piece of cake. Just eat slightly less the other six days to compensate. That's very reasonable," he says.

Research on reducing overall calorie intake, mostly in animal models, has indicated it can increase lifespan by as much as $30 \%$, according to Mark Mattson, a senior investigator for the National Institute on Aging, part of the US National Institutes of Health. In addition, he says, it can improve glu- cose regulation, reduce markers of inflammation and increase the production of ketones — which are released into the blood when the body is burning fat rather than glucose for energy. Ketones have been shown to preserve learning and memory functions and slow disease progression in the brain, says Mattson. "We are starting to understand some of the underlying mechanisms whereby fasting may benefit animals and possibly humans. We are now moving to testing some of these things in humans," he says.

There are potential harms to fasting, too. If one already has a diet poor in vitamins and protein, eating less food could lead to vitamin deficiency and muscle loss. Of course, this can be offset by taking multivitamins and doing strength training. Fasting can also lead to malnourishment if taken to an extreme. The biggest problem, though, is that it simply isn't practical for most people, says Richard Bloomer, chair of health sport sciences at the University of Memphis in Tennessee.

"I know, from working with many hundreds of people in this area, that most people aren't going to be able to do it," Bloomer says about fasting. "Wake up tomorrow and try not to eat until three in the afternoon. And then do that every day. Most people need to have a life, too. If you have kids and you wake up and want to have breakfast with them, are you going to say, 'Sorry guys, I'm not eating until three so I can lose $1 \%$ body fat.", — Roger Collier, CMAJ

A longer version of this article is available at cmaj.ca

CMAJ 2013. DOI:10.1503/cmaj.109-4437

\title{
Systematic review wins CMAJ Bruce Squires Award
}

$\mathrm{T}$ he authors of a review of evidence on the cardiovascular harm from a popular smokingcessation drug have been awarded for their contribution to health care research in Canada.

CMAJ presented the Bruce Squires Award to Dr. Sonal Singh and coauthors for their article "Risk of serious adverse cardiovascular events associated with varenicline: a systematic review and meta-analysis" (CMAJ 2011;183:135966). It was chosen from all of the papers published in the journal during 2011.

The paper examines the evidence around cardiovascular events such as heart attacks, arrhythmias, heart failure and death from cardiac arrest among people using varenicline (marketed as Chantix or Champix), a nicotine-replacement tablet.

Singh is an assistant professor of medicine and international health at Johns Hopkins School of Medicine and Public Health in Baltimore, Maryland. He coauthored the paper with Dr. Yoon Loke, the co-convenor of the Cochrane Adverse Effects Methods Group, Dr. John Spangler, professor of family and community medicine at Wake Forest University Baptist Medical Center in

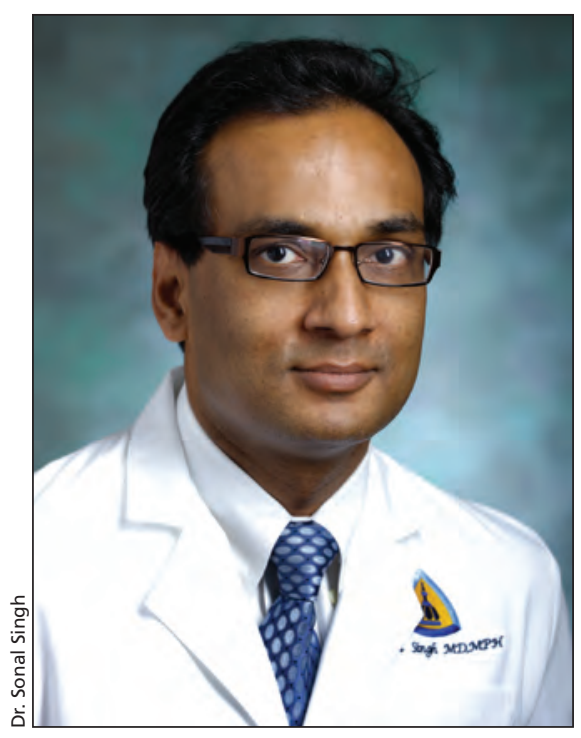

Dr. Sonal Singh and coauthors received the award for their article on the cardiovascular risks associated with varenicline.

Winston-Salem, North Carolina, and Dr. Curt Furberg, professor of public health sciences, who also works at Wake Forest.

The award honours the late Bruce Squires, who worked at CMAJ from 1984-1996, including seven years as the journal's editor-in-chief.

Squires promoted high standards of reporting and evidence-based medicine, which makes this paper a fitting choice, says CMAJ Editor-in-Chief Dr. John Fletcher.

"This is one of the few systematic reviews that has been published on the harmful effects of a drug," says Fletcher. "I think it has made a significant contribution to the medical literature."

Singh says the research has affected policy and "probably affected prescribing."

"We are now monitoring patients who are on [varenicline]," says Singh.

The United States Food and Drug Administration added cardiovascular risk warning labels on varenicline in anticipation of the paper's findings, says Singh.

The systematic review analyzed data from 14 double-blind randomized control trials with a total of 8216 participants. There were adverse cardiovascular events in $1.06 \%$ of the varenicline group compared to $0.82 \%$ in the placebo group.

"My team is probably the world's leading team in doing meta-analysis of drug safety; we've done many of them and we're actually setting standards on how to do them," he says. — Juanita Bawagan, CMAJ

CMAJ 2013. DOI:10.1503/cmaj.109-4440 